\title{
Propuesta de pliego de condiciones particulares de las reforestaciones originadas por las cortas de madera en montes con contrato administrativo
}

\author{
Arenas Ruiz, S.G. ${ }^{1}$ Izquierdo Osorio, S. ${ }^{2}$ \\ ${ }^{1}$ CFEA Becerreá (Lugo). Subdirección General de Formación e Innovación Agroforestal. \\ Consellería del Medio Rural. \\ ${ }^{2}$ Subdirección General de Biodiversidad y Recursos Cinegéticos y Piscícolas. Consellería del Medio Ambiente \\ y Ordenación del Territorio. \\ *Autor para correspondencia: santos.gabriel.arenas.ruiz@xunta.es
}

\begin{abstract}
Resumen
Uno de los principales problemas en los montes con contrato temporal de gestión pública es financiar la ejecución de la reforestación. La normativa autonómica vigente crea una sección específica y establece un fondo de mejoras, pero no regula la cuota porcentual que debe aportar la propiedad a dicho fondo, o si esta cuota alcanzará o superará la cuota mínima de reinversión en mejora y protección forestal que fija para los montes vecinales en mano común (el 40\% de todos los ingresos generados). Una opción para asegurar dicha financiación de forma inmediata es que la empresa adjudicataria del aprovechamiento ejecute a continuación la reforestación, por lo que resulta necesario establecer un pliego de condiciones para ejecutar conjuntamente ambas operaciones silvícolas. En la comunicación se establecerán las bases para la redacción de estos pliegos. Se incluye como novedad que la corta final y la reforestación se realicen dentro del mismo procedimiento administrativo, así como que la empresa adjudicataria disponga de certificación de la cadena de custodia, dado que los productos que se extraen tienen que estar avalados por un sistema de certificación forestal sostenible. La aplicación del esquema propuesto supone la intervención conjunta de la Administración forestal y de los titulares de los montes, así como un nuevo yacimiento de empleo para las empresas de ámbito local.
\end{abstract}

Palabras clave: aprovechamiento forestal, contrato público, pliego de condiciones, reforestación. 


\begin{abstract}
Summary
One of the main problems in forests with temporary contract of public management is to finance reforestations. The regional regulation creates a specific section and establishes a re-investment fund, but does not regulate the minimum percentage that the forest owner should contribute to than fund, or if this quota should reach or overcome the minimum established threshold for communal forests ( $40 \%$ of all revenues generated). An option to immediately assure the above mentioned financing is that the company in charge of the forest harvesting executes later the reforestation, being in this case necessary to establish technical specifications to execute both activities together. The fundamentals of such specifications will be proposed and discussed in this study. In such system, both the fellings and the subsequent reforestation would be included in the same administrative procedure, and the hired company would need to have certification of the chain of custody, provided that the products that are extracted have to be supported by a system of forest sustainable certification. The implementation of the proposed scheme involves the joint action by the Forest Administration and forest owners and would provide a new source of employment for local companies.
\end{abstract}

Key words: forest harvesting, public management contracts, specifications, reforestation.

\title{
1. Introducción
}

Los montes de gestión pública (MGP) en Galicia correspondían, bien a montes públicos, bien a montes privados cuyos titulares suscribieron un contrato administrativo de gestión orientado a la repoblación forestal, como los consorcios o convenios. La Ley 7/2012, de 28 de junio, de montes de Galicia regula, en su artículo 90.1, que se entiende por MGP aquellos con un contrato temporal de gestión pública (CTGP) $\mathrm{y}$, en su disposición transitoria $9^{\mathrm{a}}$.1, que los consorcios y convenios existentes a la entrada de esta ley deben transformarse en CTGP o bien cancelarse, con fecha límite de 11 de agosto del 2018.

En los consorcios la propiedad del predio (atribuida en ese momento a los ayuntamientos) sólo participaba de los beneficios netos de la madera producida, sin ser consultada sobre la gestión del monte (especie a plantar, turno de corta, tratamientos silvícolas...). Los consorcios generaron mucho empleo en el medio rural (Simón, 1952) y fueron relevantes para producir la materia prima de la incipiente industria forestal de pasta y tableros (Maraver, 1999). En ellos, todos los importes de la cuenta de gastos eran en concepto de anticipo reintegrable, con los beneficios del aprovechamiento se resarce dicho anticipo y el sobrante (beneficio neto) se reparte con la propiedad en un porcentaje fijo (Montero de Burgos, 1994).

Los convenios se limitaron a la producción de madera con especies de crecimiento rápido, consecuencia de la normativa por la que se rigen (Laborda, 2004). Las actuaciones forestales a realizar en el monte tenían que ser autorizadas por la propiedad. Los importes de la cuenta de gastos son en concepto de subvención y de anticipo reintegrable. Los beneficios (brutos) del aprovechamiento se reparten con la pro- 
piedad en un porcentaje fijado en el contrato, con la parte que va para la administración se resarce el anticipo y si hay un excedente, este revierte a la propiedad (en este sentido el porcentaje es variable).

Un aspecto esencial de los aprovechamientos madereros es que la corta final en un predio se realice conforme con los criterios de gestión forestal sostenible (GFS). Con este fin es necesario promover la regeneración tras la corta, pero en los montes con dificultades de regeneración natural (en Galicia es habitual cortar a hecho superficies pequeñas) o en los que se pretende cambiar la especie principal nos veremos obligados a reforestar, con el consiguiente coste económico.

En este contexto, los montes con un CTGP establecen una alternativa viable a la regeneración, pues, de acuerdo con el artículo 91 de la citada Ley 7/2012, de 28 de junio, como regla general, las enajenaciones de madera en corta final deberán financiar la reforestación de la superficie de corta (cuestión a tener en cuenta en momentos de reducida disponibilidad presupuestaria), además regula que la reforestación se realice en el plazo máximo de un año tras la corta y, para facilitar el cumplimiento de dicho plazo, ambas actuaciones podrán realizarse dentro del mismo procedimiento administrativo de contratación pública.

El objetivo del presente trabajo es analizar la viabilidad de realizar conjuntamente, de modo vinculado y conforme a los nuevos CTGP, actuaciones de aprovechamiento maderero y la reforestación de las áreas de corta final. Adicionalmente, se propondrá la estructura básica de un pliego de condiciones en el que la corta final y su reforestación se realizan en un mismo procedimiento administrativo, asimismo, se discutirán los posibles beneficios que el sistema propuesto puede presentar en la economía rural y en las empresas de ámbito local de aprovechamiento y servicios forestales.

\section{Condicionantes actuales de la propuesta}

La Ley 7/2012, de 28 de junio, en su título X (Fomento forestal) regula los CTGP y establece que, para los montes con este tipo de contrato, la GFS se realizará a través de un proyecto de ordenación forestal, inscrito en el Registro Gallego de Montes Ordenados, y estará evaluada al menos por un sistema de certificación forestal reconocido internacionalmente y validado por las correspondientes entidades de certificación. Siempre se mantendrá informada a la propiedad (colectiva en la mayoría de los casos) de la ejecución de las actuaciones recogidas en el proyecto de ordenación y de las incidencias que puedan surgir.

El importe de las inversiones en obras y servicios realizados con cargo a los CTGP se compensará con la aplicación de unas determinadas cuotas porcentuales (no fijadas por la ley), con cargo a los ingresos obtenidos del monte (aprovechamientos forestales, expropiaciones, derivados de actos de disposición voluntaria...).

En relación a los aprovechamientos madereros, su ejecución en MGP se regirá mediante pliegos de prescripciones técnicas (artículo 90.2 de la Ley 7/2012, de 28 de junio). El pliego, que rija tanto el aprovechamiento maderero como la posterior 
reforestación, debe ajustarse a la legislación sobre contratos de las Administraciones Públicas, RDL 3/2011, de 14 de noviembre, y RD 1098/2001, de 12 de octubre. Como normas subsidiarias son de aplicación las resoluciones de la Dirección del ICONA por las que se aprueban el pliego general de condiciones técnico facultativas para regular la ejecución de disfrute en montes a cargo del ICONA, de 24.04.1975, y los pliegos especiales de condiciones técnico facultativas para la regulación de los aprovechamientos maderables y de corcho en los montes a cargo del ICONA, de 06.05.1975.

Las empresas adjudicatarias de estas actuaciones forestales (explotación y reforestación) deben: estar inscritas en el Registro de Empresas del Sector Forestal (artículo 102 de la Ley 7/2012, de 28 de junio), poseer la certificación de la cadena de custodia (los productos a extraer deben estar avalados por un sistema de certificación forestal sostenible (CFS) -artículos 123.3 y 105.4 de la Ley 7/2012-) y tener la capacidad de ejecutar las obras tanto con maquinaria ligera (motosierras, motodesbrozadoras y motoahoyadoras) como pesada (cosechadoras, autocargadores y tractores forestales); además, deben de suministrar anualmente a la Consellería competente en materia de montes, a efectos estadísticos, información relativa a su actividad (artículo 103.1 de la Ley $7 / 2012$ ).

\section{Propuesta de contenido del pliego}

A continuación se propone el contenido mínimo, conforme con la legislación vigente, que debe presentar el pliego de prescripciones técnicas para la corta a hecho seguida de la posterior reforestación.

\subsection{Descripción de la actuación}

La enajenación llevaría incluida la posterior reforestación de la superficie de corta por parte de la empresa adjudicataria. Debería fijarse el plazo máximo de ejecución de la obra (incluida la extracción de los productos leñosos, gestión de restos y repoblación). Ya se ha indicado que la reforestación debería realizarse en un plazo máximo de un año tras la corta.

El pliego debe incluir la información del monte: denominación (nombre), número del CTGP, pertenencia (propiedad) y localización (parroquia, término municipal y distrito forestal); así como los datos del rodal de actuación: descripción de los límites, situación en plano topográfico, superficie (hectáreas) y sistema de certificación forestal. El plano se georreferenciará con las delimitaciones necesarias para la correcta ubicación y realización de los trabajos.

Las especificaciones de la enajenación deberán incluir: núm. de lote, especie, núm. de pies, volumen estimado $\left(\mathrm{m}^{3}\right)$, tasación (precio unitario $€ / \mathrm{m}^{3}-\mathrm{e}$ importe total $€-$ ), tasa de corta, garantía provisional, procedimiento de adjudicación (por subasta, procedimiento negociado o enajenación directa -artículo 90.1 de la Ley 7/2012-), sistema de determinar el volumen de corta ("a riesgo y ventura" o "a re- 
sultas con liquidación final"), situación de los pies en el monte (en pie), control de las operaciones (contada en blanco, medición -en volumen o en peso-).

Las especificaciones de la reforestación afectarían a: especie, material origen y categoría del material forestal de reproducción, marco de plantación, eliminación de los restos de corta, preparación del suelo, fertilización y protección de la planta si procede y plantación.

En las especificaciones económicas se fijará: IVA, gastos de publicidad (por cuenta de la empresa adjudicataria), cuenta bancaria del titular del monte. Debe considerarse que la oferta económica, que plantee la empresa, tendrá que basarse no solo en la tasación de la madera sino también en la estimación de los costes de reforestación, que tendría que asumirlos posteriormente a la corta.

\subsection{Proceso y formalización de la adjudicación}

Quien licita deberá presentar la documentación habitual en el procedimiento de subasta, (sobre con la oferta económica y sobre con los datos de quien licita). La formalización de la adjudicación supone la obtención de la licencia de corta una vez que ésta sea firme y se le haya notificado formalmente, la constitución de la garantía definitiva, el abono del importe del aprovechamiento (IVA incluido) y la firma del pliego de condiciones particulares en todas sus hojas.

Tras expedir la licencia de corta se levantará el acta de entrega, en la que deben reflejarse además de las características del aprovechamiento las labores de reforestación, así como sus medidas de control. La ejecución de la actuación será controlada e inspeccionada por personal del Distrito Forestal o/y del Servicio Provincial de Montes. La fianza definitiva se devolverá una vez realizado el reconocimiento final, tanto del aprovechamiento como de la reforestación, conforme con las condiciones establecidas en el pliego y en el acta de entrega.

\subsection{Prescripciones técnicas particulares del aprovechamiento $y$ de la reforestación}

El aprovechamiento forestal incluye las operaciones de apeo, desramado, tronzado, saca, carga y transporte. Se establecerán las condiciones de apeo, saca y transporte que se consideren necesarias, siendo relevante, para planificar la futura repoblación, las posibles actuaciones de destoconado y la gestión imprescindible de los restos de corta generados.

En Galicia no es habitual destoconar tras las cortas de madera, salvo por problemas sanitarios, por sustitución de especies rebrotadoras o por aprovechamiento energético. Sólo en estos casos, en los pliegos de condiciones, debe quedar claramente establecida la necesidad de destoconar, dado que esta operación supone un coste importante y condiciona las posteriores actuaciones de preparación del suelo.

En cuanto a la repoblación, el pliego de condiciones debería incluir en sí mismo un proyecto sucinto de los trabajos a desarrollar.

Debe considerarse que el tratamiento de los restos de corta (raberones menores 
de $7 \mathrm{~cm}$ de diámetro y leñas menores de $50 \mathrm{~cm}$ de longitud), en caso de no ser extraídos, deberán ser tratados in situ, triturándolos si la labor es mecanizable (Quindous, 1996) o picándolos de forma manual; otras alternativas justificables son el acordonado según curvas de nivel (Arenas, 1998) o la solicitud de una autorización de quema. La Ley 7/2012, de 28 de junio, en su artículo 95.1 regula, para los montes de gestión privada, la trituración in situ o la extracción para su aprovechamiento, salvo justificación técnica de no poder ejecutarlo.

Las alternativas para la preparación del suelo son variadas: subsolado, ahoyado con rejón modificado, ahoyado con miniretros o con retroarañas (Serrada, 2000), preparación de casillas picadas con motoahoyadora o incluso miniretros o ahoyado manual. Su ejecución estará condicionada adicionalmente por la presencia de tocones.

La plantación habitualmente se realizará de forma manual, con plantamón -plantas a raíz desnuda- o con bastón plantador - plantas con cepellón-. Un aspecto relevante es la admisión de la planta y el cumplimiento de la normativa sobre materiales forestales de reproducción y documentos de acompañamiento. En concreto deberá considerarse el D 220/2007, de 15 de noviembre, por el que se crea el sistema oficial para el control de la producción y comercialización de los materiales forestales de reproducción y, supletoriamente, el RD 289/2003, de 7 de marzo (requisitos de origen y calidad exterior) y el RD 58/2005, de 21 de enero (requisitos sanitarios).

En las labores mecanizables y durante la época de peligro alto de incendios, los tractores, máquinas y vehículos de transporte pesado deben estar dotados de dispositivos de retención de chispas y de dispositivos antillamas en el tubo de escape, además deben de ir provistos de equipamiento para la extinción de incendios (artículo 39.1 de la Ley $3 / 2007$, de 9 de abril, de prevención y defensa contra incendios forestales de Galicia).

Es importante la realización de parcelas de contraste, en presencia de la dirección de obra facultativa, en el programa de pruebas que se establece para controlar la ejecución de la repoblación.

\subsection{Medidas complementarias en relación a incendios forestales y residuos}

La empresa adjudicataria deberá cumplir las condiciones habituales establecidas en los pliegos de condiciones, referidos a aprovechamientos madereros y a repoblaciones, encaminadas a la prevención y extinción de los incendios forestales y a la adecuada gestión de los residuos dentro del monte.

La combinación de las actuaciones de corta y reforestación conlleva un aumento del personal y de maquinaria a disposición del distrito forestal en el monte. Ello ofrece ventajas tanto en la detección como en la extinción o prevención de incendios.

En relación con los residuos (no tienen tal consideración los restos forestales producidos) causados durante las actividades, la empresa adjudicataria cumplirá los requisitos exigidos por la normativa vigente y por los sistemas de certificación fores- 
tal. La propuesta de vinculación de ambos tipos de trabajo resulta positiva, pues supone que son realizados por la misma empresa, la cual debe estar avalada por un sistema de CFS, y conforme con los criterios de GFS.

\subsection{Seguridad y salud laboral}

La empresa adjudicataria cumplirá la legislación vigente en materia de prevención de riesgos laborales. Los equipos de trabajo deberán poseer la información y la formación, los equipos de seguridad homologados y demás requerimientos preceptivos. La propuesta de vinculación de ambos tipos de trabajo supone una cierta complejidad para las empresas, pues los requisitos de seguridad y salud laboral en el ámbito de los aprovechamientos madereros difieren de los correspondientes en la reforestación, lo que posiblemente conllevaría a contar con cuadrillas y tractoristas especializados en ambos tipos de trabajo.

\section{Resultados}

Los nuevos CTGP se suscribirán mayoritariamente con propiedades germánicas y romanas proindiviso (titulares de gran parte de los antiguos consorcios y convenios) en las que no están garantizadas la sostenibilidad económica, social y ambiental. En consecuencia, los montes que proporcionan rentabilidad económica tendrán muy posiblemente una gestión privada.

La Ley 7/2012, de 28 de junio, en su artículo 124.1, establece un fondo de mejoras para realizar inversiones de carácter forestal, con una sección específica para montes con CTGP. Asimismo, en su artículo 124.6, dispone que los titulares de los MGP depositarán la cuota porcentual fijada en el contrato suscrito con cargo a los ingresos obtenidos (aprovechamientos, expropiaciones, derivados de actos de disposición voluntaria...), hasta satisfacer las cantidades invertidas por la Administración forestal en el monte en concepto de anticipos reintegrables y gastos por servicios de gestión, pudiendo tener como consecuencia la extinción automática del contrato. Esta ley no indica cómo distribuir el fondo de mejoras para asegurar que las aportaciones depositadas reviertan en el monte con CTGP que generó el aprovechamiento.

La fórmula propuesta en este trabajo (vinculación del aprovechamiento a la reforestación) supondrá una condición a añadir en los nuevos CTGP. La propiedad recibirá los ingresos derivados de los aprovechamientos disminuidos en los gastos de la repoblación y, adicionalmente, estará obligada a cumplir los porcentajes de reinversión en mejora (mínimo el 40\% para propiedades colectivas) que fija la ley autonómica de montes. En el caso de que estos ingresos excedieran lo presupuestado para ejecutar los trabajos anuales programados en el correspondiente instrumento de gestión forestal, el indicado porcentaje podría reducirse.

Si los nuevos CTGP se orientan a montes protectores, a montes descapitalizados de propiedad colectiva y a montes particulares con condicionantes importantes de 
gestión (masas de alto valor genético, formaciones significativas de frondosas o montes con especiales dificultades para su puesta en valor), la ejecución inicial de la forestación tendría que financiarse, en una cierta proporción, como anticipo reintegrable con cargo al fondo de mejoras o a la Administración forestal.

La ventaja del sistema propuesto es evidente en cuanto a que los ingresos proporcionados por el aprovechamiento cubren las inversiones a realizar en reforestación, ésta se realiza en un plazo reducido tras la corta y ambos en un único procedimiento administrativo. La alternativa clásica a este sistema es condicionar los gastos de reforestación a las obligaciones de reinversión a que esté sujeta la propiedad.

El sistema propuesto requiere un pliego de condiciones particulares que regule las operaciones de corta y reforestación, condicionando tanto los materiales a emplear como los procesos a seguir y la calidad de ejecución final. Es evidente que dicho pliego de condiciones debe determinar de forma precisa y concreta las necesidades, expectativas y limitaciones de la Administración forestal.

Una complejidad añadida a esta propuesta es el hecho de que la tipología de trabajos que debe abordar la empresa adjudicataria es muy amplia, aunque en nuestra opinión supondría una mayor profesionalización del sector y una vinculación de las empresas de aprovechamiento a la realización de obras de repoblación y mejora forestales.

Dadas las dimensiones de estas obras (en la mayoría de los casos la superficie de corta por aprovechamiento es inferior a las 10 hectáreas), el aprovechamiento y la posterior repoblación podrán ser realizados por empresas de ámbito local -forma empresarial concordante con la "empresa de economía social" (Ballestero, E.; 1.990)-, lo que probablemente redundaría en un aumento del empleo forestal en áreas rurales (criterio 6 paneuropeo).

Estas empresas de ámbito local responden a la implantación de actuación en materia de responsabilidad social empresarial (RSE) en la cadena de valor de la madera, puesto que se ponen en marchas actuaciones responsables y estratégicamente competitivas. Muchas de ellas han ideado, en los últimos años, innovaciones tecnológicas conforme a las necesidades que surgen (por ejemplo el cabezal desbrozadorahoyador en la retroaraña, rejón modificado ahoyador).

Desde finales del siglo XX, un reducido número de empresas de ámbito local puede ejecutar trabajos mecanizados debido a que han ampliado sus inversiones en activos fijos productivos, en parte financiados con las ayudas para creación y mejora de empresas de aprovechamientos forestales en el marco del Plan de desarrollo rural sostenible.

\section{Conclusiones}

La Administración forestal debería considerar, en un futuro inmediato -tras la redacción del pliego de condiciones-, la conveniencia de que la enajenación de la madera y los trabajos de reforestación se realicen dentro del mismo procedimiento administrativo de contratación pública, en el marco de los nuevos CTGP. 
Esta propuesta (vinculación del aprovechamiento a la reforestación dentro del mismo procedimiento administrativo) podría agilizar la tramitación administrativa que permite realizar aprovechamientos madereros y repoblación conjuntamente. Además es compatible con la existencia del fondo de mejoras y las cuotas de reinversión obligatorias. Igualmente puede constituir un aliciente para que la propiedad se interese por los CTGP (al asegurar la persistencia de la masa evitando la financiación).

La propuesta también puede ser positiva para fomentar la figura de la empresa de ámbito local, capaz de potenciar los intereses socioeconómicos de los propietarios y propietarias, que constituyen un nuevo yacimiento de empleo.

\section{Bibliografía}

Arenas, S. 1998. Método de repoblación manual en fajas. Mesa nº 2. I Congreso forestal Hispano-luso. Pamplona.

Ballestero, E. 1990. Economía social y empresas cooperativas. Alianza Editorial. Madrid.

Laborda, E. 2004. Alrededor de la singular naturaleza jurídica de los convenios para la repoblación forestal. UNED. Boletín de la Facultad de Derecho 25, 319-342.

Maraver, E. 1999. Los consorcios y la administración: una historia que no debe ser interminable. Montes 57, 61-66.

Montero de Burgos, J. 1994. Actualizar los antiguos consorcios. Revista Forestal Española 10, $6-10$.

Quindous, M. 1996. Estudio de la mecanización empleada en los procesos de desbroce y ahoyado forestal. Proyecto fin de carrera. Escola Politécnica Superior de Lugo. Universidad de Santiago de Compostela. Inédito.

Serrada, R. 2000. Apuntes de repoblaciones forestales. FUCOVASA. Madrid.

Simón, E. de 1952. Repoblaciones en consorcio. Montes 46, 241-245. 
